\title{
Intestinal Malrotation with Midgut Volvulus leading to Small Bowel Obstruction in a Young Adult: A Case Report
}

\author{
Junaid Zia Hashmi ${ }^{1}$, Talha Kareem ${ }^{1}$, Masood ur Rauf Khan ${ }^{2}$ \\ ${ }^{1}$ Post Graduate Resident, Nishter Hospital Multan, Pakistan \\ ${ }^{2}$ Professor, Head of Department, Surgical Unit IV, Nishter Hospital Multan, Pakistan
}

\begin{abstract}
The developing intestine rotates around the superior mesenteric vessels during physiological herniation from $6^{\text {th }}$ to $10^{\text {th }}$ weeks of embryogenesis. Intestinal malrotation leading to midgut volvulus and small bowel obstruction is a rare condition. Patients who develop this condition usually present in the first year of their life. Intestinal malrotation is characterized by right sided duodenojejunal junction, caecum in left iliac fossa and a narrow mesentery. Ladd's procedure is the treatment of choice which is done to restore the normal anatomy. We report the case of an 18-yearold male patient, who presented with intestinal malrotation in emergency department of Nishtar Hospital, Multan. It is an uncommon age for presentation of this anomaly. The patient had fecal peritonitis due to caecal perforation. He underwent Ladd's procedure in which ileocolostomy was made after right hemicolectomy. The patient had an uneventful recovery after the surgery and had no complaints on follow up visit one week after the discharge.

Key Words: Intestinal malrotation, Midgut volvulus, Right hemicolectomy, Small bowel obstruction Correspondence: Article info:

Junaid Zia Hashmi

Received: March 23, 2020

Email: juneehashmi@yahoo.com Accepted: June 12, 2020

Cite this case report: Hashmi JZ, KareemT, Khan MR. Intestinal Malrotation with Midgut Funding Source: Nil Volvulus leading to Small Bowel Obstruction in a Young Adult: A Case Report. I Islamabad Med

Conflict of Interest: Nil

Dental Coll. 2020; 9(2):.149-152. Doi: 10.35787/jimdc.v\%vi\%i.520
\end{abstract}

\section{Introduction}

Obstruction of intestines is either due to a mechanical or a dynamic cause. Among adults, hernia and adhesions are the two most common causes. ${ }^{1}$ Presentation of small bowel obstruction due to intestinal malrotation usually presents during infancy, therefore cases appearing later in life cause difficulties in diagnosis. ${ }^{2}$ During normal fetal development midgut rotates around the superior mesenteric artery up to $270^{\circ}$ in anticlockwise direction. Following this rotation, the bowels return to the abdominal cavity, with fixation of the duodenojejunal loop to the left of the midline and the cecum in the right lower quadrant. ${ }^{3}$

Congenital malrotation develops when there is either partial or no rotation of the returning gut.
Interruption of normal intestinal rotation and fixation can occur at a wide range of locations which leads to different acute and chronic presentations of the disease. ${ }^{4}$ The most common presentation is incomplete rotation leading to midgut volvulus in infants and requires immediate surgical intervention. The clinical condition of small bowel obstruction due to congenital malrotation of gut was first described by William $E$ Ladd, a pediatric surgeon, who also described the corrective surgical procedure in $1932 .{ }^{4}$ This procedure still remains the cornerstone of practice nowadays.

According to a study, $90 \%$ of patients who present with malrotation are infants. ${ }^{2}$ Patients who remain 
asymptomatic in initial years, present as a case of intestinal obstruction later in their lives and are frequently misdiagnosed. Intestinal malrotation leading to mid gut volvulus in older age group accounts only for $0.2-0.5 \%$ of cases..$^{5-7}$

Here, we report an unusual case of caecal volvulus due to intestinal malrotation leading to caecal perforation in an 18-year-old male patient.

\section{Case Report}

An 18-year-old male, resident of Dera Ismail Khan, Khyber Pakhtunkhwa, Pakistan presented to the emergency department of Nishtar Hospital Multan on $24^{\text {th }}$ August, 2019. He complained of generalized abdominal pain for the last 7 days, and vomiting, constipation and abdominal distention for 02 days. There was no history of fever, weight loss, or any previous abdominal surgery. There was no history of pulmonary tuberculosis. Patient was a nonsmoker and not addicted to anything. Systemic history was non-significant.

Clinical examination revealed a lean young boy of normal built and height. Pulse rate was 108/min, blood pressure $100 / 70 \mathrm{mmHg}$, respiratory rate $22 / \mathrm{min}$ and temperature $101^{\circ} \mathrm{F}$. Abdomen was distended, tense, mildly tender, hernial orifices were intact and no lymph nodes were palpable. Bowel sounds were absent. Digital rectal examination revealed empty rectum with no palpable mass.

Laboratory workup showed hemoglobin $14.2 \mathrm{~g} / \mathrm{dl}$ and TLC count $13,000 / \mathrm{mm}^{3}$. Renal and liver function tests were normal. Abdominal erect X-ray showed dilated jejunal and ileal gut loops. Chest $X-$ ray was unremarkable. Ultrasound abdomen revealed gut loops with absent peristalsis and free fluid in the peritoneal cavity.

Exploratory laparotomy was done, which revealed intestinal malrotation leading to midgut volvulus, caecal perforation and fecal peritonitis (Figure 1).
Ladd's procedure was carried out to restore the normal anatomy. Prolonged obstruction caused caecal perforation, so right hemicolectomy was done and an ileocolostomy was made. Patient was allowed liquid and soft diet on $3^{\text {rd }}$ post-operative day. The patient had an uneventful recovery after the surgery and had no complaints on follow up visit one week after the discharge.

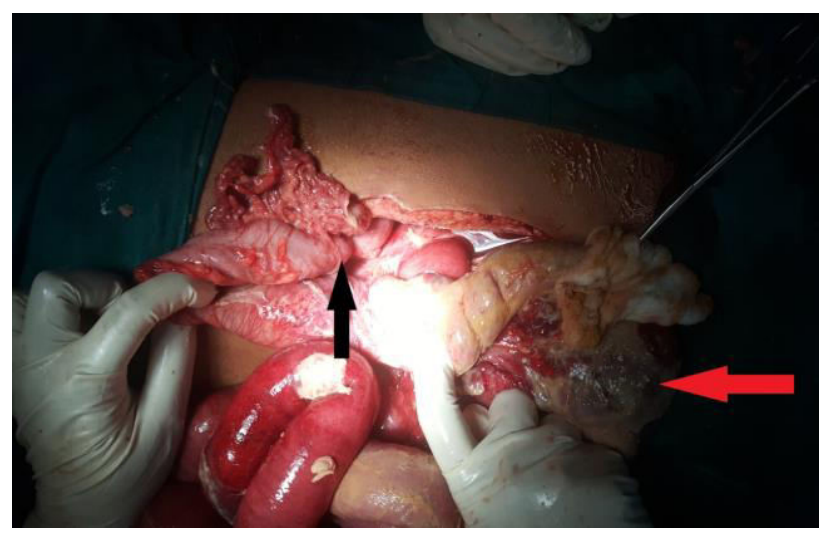

Figure 1: Picture showing intestinal malrotation. Dudenojejunal junction shown on right side (black arrow) and ileocecal junction on left side (red arrow).

\section{Discussion}

During the sixth week of embryological development, the rapid growth of the midgut renders it too large to be accommodated in the abdominal cavity. This leads to physiological herniation of the gut loops into the umbilical cord. Subsequently a 270-degree counterclockwise rotation of the gut occurs around the superior mesenteric artery. Malrotation of gut is a congenital defect caused by the incomplete or absent rotation of the gut. This leads to abnormal placement of the gut and various forms of clinical presentations.

Midgut volvulus is more common in the first few days of life with the presenting complaint of bilious vomiting. Any irritable child with bilious emesis should be immediately evaluated for volvulus due to malrotation of midgut. The incidence of midgut volvulus due to intestinal malrotation is rare in 
adults with the rate approximately being $0.2 \%$ $0.5 \%{ }^{3,8}$ This patient presented to the emergency with the presenting complaints that were suggestive of acute intestinal obstruction. There was generalized abdominal pain for past one week with abdominal distension and absolute constipation for last 2 days. This presentation is quite different to that of an infant, who presents with irritability and bilious vomiting with bloody stools, if left untreated. This can further progress to circulatory shock leading to death of the infant. ${ }^{9}$ Adults on the other hand can have an acute or a chronic presentation. Usually a chronic presentation is more common and results in frequent visits to the hospital with crampy abdominal pain and vomiting not subsiding with conservative measures, thus posing a challenge for the surgeon. ${ }^{3}$ Acute presentation, though uncommon, may present with intestinal ischemia and gangrene which has disastrous consequences. ${ }^{10}$ In adults, the diagnosis requires a high index of suspicion because timely recognition is the key to survival.

Plain abdominal radiographs have not been helpful and the condition is best diagnosed by upper gastrointestinal series that show the displacement of duodenojejunal flexure to the right. ${ }^{9}$ The findings suggestive of malrotation of gut include a corkscrew effect indicating a volvulus or a complete duodenal obstruction. In other cases, the entire bowel presents on the right side. In addition, there is a whirled appearance of the vessels entering the volvulus, hence named as the "whirlpool" sign. ${ }^{6}$ Recent trends are suggesting an increasing use of CT scan with or without upper GI contrast studies with diagnostic accuracy ranging up to $80 \% .{ }^{11,12}$

Once the patient has presented to the hospital and has been diagnosed, surgical intervention is the best option with Ladd's procedure as the treatment of choice. The procedure involves division of any bands between cecum and abdominal wall and between duodenum and terminal ileum. This procedure has the benefit of splaying the superior mesenteric artery and subsequently helping in bringing duodenum in right lower quadrant and cecum in left lower quadrant. Procedure is concluded with appendectomy to avoid any confusion in diagnosis later in life. ${ }^{8}$ However, this procedure is not done if there is severe ischemia at presentation. The volvulus is reduced without Ladd's procedure and reassessed after 24-36 hours.

\section{Conclusion}

Small bowel obstruction due to congenital malrotation of gut usually presents during the first year of life. Although obstructed hernia and adhesions are the most common causes of bowel obstruction in adults, congenital malformation should be kept in mind as a differential diagnosis. This is important as early surgical intervention can decrease the morbidity and mortality.

\section{References}

1. Rami Reddy SR, Cappell MS. A Systematic Review of the Clinical Presentation, Diagnosis, and Treatment of Small Bowel Obstruction. Curr Gastroenterol Rep. 2017; 19(6): 28. Doi:10.1007/s11894-017-0566-9

2. Vukie Z. Presentation of intestinal malrotation syndromes in older children and adults: Report of three cases. Croat Med J. 1998; 39(4): 455-57.

3. Bhatia S, Jain S, Singh CB, Bains L, Kaushik R, Gowda N S. Malrotation of the Gut in Adults: An Often Forgotten Entity. Cureus. 2018; 10(3): e2313. Doi: 10.7759/cureus.2313

4. Hmadeh H, Saliba C, Raka M, Farhat HA, Dabbous A, Diab $S$, et al. An Unusual Case of Intestinal Malrotation Causing Duodenal Obstruction by a Looped Appendix. Am J Case Rep. 2018; 19: 136265. Doi: 10.12659/AJCR.913039

5. Fung AT, Konkin DE, Kanji ZS. Malrotation with midgut volvulus in an adult: a case report and review of the literature. J Surg Case Rep. 2017; 2017(5): rjx081. Doi: 10.1093/jscr/rjx081

6. Low SF, Ngiu CS, Sridharan R, Lee YL. Midgut malrotation with congenital peritoneal band: a rare 
cause of small bowel obstruction in adulthood. BMJ Case Rep. 2014; 2014: bcr2013202690. doi:10.1136/bcr-2013-202690

7. Torres A., Ziegler M. Malrotation of the intestine. World J Surg. 1993; 17: 326-331.

8. Kache SA, Sale D, Yusuf N, Makama JG. Malrotation with midgut volvulus and bowel gangrene in a 45year-old man. Arch Med Surg. 2017; 2: 29-31

9. Smink DS. Schwartz's principles of surgery. Ann Surg. 2015; 261(5): 1026.
10. Asma H, Khalid R, Abdelouahed L, Benasser $F$. Midgut Volvulus: Acute Abdomen in an Adult Patient. JUCMS. 2017; 14: 246-247. Doi: 10.17265/1548-6648/2017.06.005

11. Narayanasamy SN, Manoharan GV, Padmanaban N. Reverse rotation of gut with small bowel volvulus. Int Surg J. 2015; 2: 295-9.

12. Lai YK, Mahmood RD. Clinics in diagnostic imaging (168). Singapore Med J. 2016; 57(5) 274-78. Doi:10.11622/smedj.2016093. PMID: 27212130 